Cell Research (1999), 9, 201-208

\title{
The developmental fate of green fluorescent mouse embryonic germ cells in chimeric embryos
}

\author{
XU XIn ${ }^{1, *}$, Yong Shen YU ${ }^{1, *}$, Hsiao Chien TSUNG ${ }^{1}$, \\ Sumio SUGANO ${ }^{2}$, Yuan Chang YAN ${ }^{1, * *}$ \\ 1. Shanghai Institute of Cell Biology, Chinese Academy of \\ Science, Shanghai 200031, China \\ 2. Department of Virology, The Institute of Medical Science, \\ The University of Tokyo, Tokyo, Japan
}

\begin{abstract}
Primordial germ cells (PGCs), as precursors of mammalian germ lineage, have been gaining more attention as a new resource of pluripotent stem cells, which bring a great possibility to study developmental events of germ cell in vitro and at animal level. EG4 cells derived from 10.5 days post coitum (dpc) PGCs of 129/svJ strain mouse were established and maintained in an undifferentiated state. With an attempt to study the differentiation capability of EG4 cells with a reporter protein: green fluorescence protein, and the possible application of EG4 cells in the research of germ cell development, we have generated several EG4-GFP cell lines expressing enhanced green fluorescence protein (EGFP) and still maintaining typical characteristics of pluripotent stem cells. Then, the differentiation of EG4-GFP cells in vitro as well as their developmental fate in chimeric embryos which were produced by aggregating EG4-GFP cells to 8-cell stage embryos were studied. The results showed that EG4 cells carrying green fluorescence have a potential use in the research of germ cell development and other related studies.
\end{abstract}

Key words: Embryonic germ cell, chimera, EGFP, gene transfection.

\footnotetext{
* Authors with equal contribution to the work

** Corresponding author, e-mail: ycyan@sunm.shcnc.ac.cn
} 
The developmental fate of green fluorescent mouse embryonic germ cells in chimeric embryos

\section{INTRODUCTION}

In vertebrates, the development of germ line which arises from totipotent precursor cells is a continuous process from the time that primordial germ cells (PGCs) can be identified during early development until the gametes are released in adults. In mouse, PGCs are thought to arise as a small population of epiblast cells at about 7 days post coitum (dpc). By 7.5 dpc, PGCs which are identified by abundant levels of endogenous alkaline phosphatase (AKP) can be detected in the extra-embryonic mesoderm, just posterior to the primitive streak near the base of allantois. During subsequent $4-5 \mathrm{~d}$ of development, PGCs migrate along the hindgut to the gonad anlage, and the PGC number increases dramatically to around 25,000 cells when PGCs stop dividing at $13.5 \mathrm{dpc}$. Then PGCs enter a sexual differentiation phase followed by a gametogenesis phase. Although eggs and sperm are highly differentiated in structure and function, they all have the developmental potential to combine and give rise to a new organism[1].

For a decade or so the major hindrance to our understanding of the development of mammalian germ line was the inability to obtain long term culture of PGCs in vitro. More recently, PGCs have become a new source of pluripotent stem cells since they can transform into embryonic germ cells (EG cells) when cultured on feeder layer cells in the presence of stem cell factor (SCF), leukemia inhibitory factor(LIF) and basic fibroblast growth factor(bFGF). Once established, EG cells can be maintained in an undifferentiated state without added bFGF and SCF, but still need feeder cells or LIF in culture system[2-4]. We have established five murine EG cell lines derived from PGCs of 8.5, 10.5 dpc embryos. Under appropriated conditions, EG cells formed embryonic bodies in suspension culture and teratocarcinomas containing different types of differentiated cells when injected into nude mice[4]. EG cells could give rise to germline transmission chimeric mice after their introduction into a host blastocyst and transfer into a recipient female[5],[6]. These results showed that EG cells share many characteristics of morphology, growth patterns and differentiation capability with embryonic stem (ES) cells derived from inner cell mass of early embryos. The derivation of totipotent stem cell lines from stages of development other than preimplantation embryos is of interest since it may facilitate the establishment of such lines from other species in which it is difficult to culture or establish ES cell lines from embryos of preimplantation stages.

In an attempt to exploit potential use of EG cells in the development of germ cell and transgenic research, we have transfected one of our EG cell lines with plasmid pCE-EGFP and established several green EG cell lines (EG4-EGFP) expressing enhanced green fluorescence protein (EGFP), but still retaining typical phenotype of pluripotent stem cells in vitro. Here, we will report the distribution of EG cells in chimeric embryos produced by the aggregation of EG4-GFP cells to 8-cell stage embryos. 


\title{
MATERIALS AND METHODS
}

\author{
EG4 cell line[4]
}

EG4 cells derived from 10.5 dpc embryos of $129 \mathrm{svJ}$ strain mouse were maintained on the feeder layer cells of mouse embryonic fibroblast (MEF) in DMEM medium containing $15 \%$ FCS, $2 \mathrm{mM}$ glutamine, $1 \mathrm{mM}$ sodium pyruvate, $1 \mathrm{mM}$ nonessential amino acid, $0.1 \mathrm{mM}$ 2-mercaptoethanol.

\section{EG4-GFP cell line}

The plasmid pCE-EGFP was constructed by Kim et al[7] in which EGFP gene is driven by the EF321 promoter together with the CMV enhancer. The plasmid contains a selectable marker, neo. Plasmid DNA was extracted with QiaGen kit. Fortyeight hours after transfection with Lipofectamine reagent, EG4 cells were selected in $0.4 \mathrm{mg} / \mathrm{ml} \mathrm{G} 418$ containing medium with a change on every other day. After 10 days or so, G418-resisit clones were picked up randomly, and expanded those with observable green marker protein under Olympus microscope with fluorescent optics. Eleven positive clones with GFP expression were expanded further and frozen.

\section{In vitro differentiation}

A $100 \mathrm{~mm}$ dish were preloaded by $1 \%$ Agar, EG4 cell suspensions with a density of $2 \times 10^{6}$ were cultured on Agar in DMEM containing $10 \%$ FBS with no LIF addition. After 3-4 d, embryoid bodies were recultured on gelatin-treated 24-well plate, and differentiated cells were growing out within 1-2 d, observed under fluorescence optic.

\section{Collection of embryos[8]}

C57BL/6J females were superovulated with 7.5 units of pregnant mare serum gonadotropin, followed $46-48 \mathrm{~h}$ later by 7.5 units of human chrionic gonadotropin and were mated with males of the same strain. In the next morning females with vaginal plugs were designated as $0.5 \mathrm{~d}$ of pregnancy $(0.5 \mathrm{dpc})$. To recover eight-cell embryos, the oviducts were flushed with M2 medium at $2.5 \mathrm{dpc}$. Zona pellucida were removed by 5 to 10 sec exposure to acidified Tyrode' s.

\section{Aggregation of EG4-GFP cells with embryos of 8-cell stage[8],[9]}

MEF cells were seeded at a density of $2.5-4 \times 10^{5}$ cells per well (4-well plate) in DMEM medium containing $10 \%$ NCS. $1 \%$ Agar in DMEM medium containing $10 \%$ NCS was plated onto feeder layer after medium was removed. Some holes were made in agar layer with fine needle under microscope, and 8-cell stage embryos were placed into holes in M16 medium supplemented with $10 \% \mathrm{FCS}$. After incubation at $37^{\circ} \mathrm{C}$ for $1 \mathrm{~h}$, a single group of several EG cells was placed into each hole to make two different kinds of aggregates: a single 8-cell stage compacted or uncompacted embryo with EG 
The developmental fate of green fluorescent mouse embryonic germ cells in chimeric embryos

cells and two embryos with EG cells in the middle as sandwiches, then make sure that EG cells remained sticking to embryos. Aggregates were cultured for 30-36 h while most of them developed into blastocysts. The location of EG-GFP cells in chimeric embryos was examined under fluorescence microscope. Some manipulated embryos were transferred to the uterine horns of two pseudopregnant F1 (ICR $\times$ Balb/c) female on 2.5 dpc.

\section{Microinjection of EG4-GFP cells into 8-cell stage embryos[10]}

A group of 1-10 EG4-GFP cells was microinjected into the perivitelline space of 8cell stage embryo, and the host embryos were cultured in M16 supplemented with $10 \% \mathrm{FBS}$ overnight at $37^{\circ} \mathrm{C}, 5 \% \mathrm{CO}_{2}$. The chimeric blastocysts were transferred to the uteri of pseudopregnant F1 (ICR\&3215; Balb/c) female on $2.5 \mathrm{dpc}$.

\section{RESULTS AND DISCUSSIONS}

\section{Generation of green EG4 cells}

EGFP expressing EG4 cell lines (EG4-GFP) were generated by the transfection of pCE-EGFP expressing vector containing a selectable marker. The G418 resistant colonies were expanded and showed strong green fluorescence under microscope equipped with fluorescence optic (Fig 1). EG4-GFP cells showed typical characteristics of pluripotent stem cell and could be cultured on MEF layers as nest-like colonies with high AKP activity. In the absence of feeder layer cells, EG4-GFP cells

Fig 1.

Expression of EGFP in a colony of EG4-GFP cells, showing green fluorescence. $(\times 200)$

Fig 2.

An embryoid body derived from EG4-GFP cells showing bright green fluorescence. $(\times 100)$

Fig 3.

Differentiated cells (arrow indicated) grew out from embryoid bodies plated onto 24-well plate (a: natural light); green fluorescence could be observed under UV light (b). $(\times 100)$

Fig 4.

a: EG4-GFP cells attached to an uncompacted 8-cell stage embryo, with regular optics. $(\times 400)$

b: The same field of Fig 4 a, but with fluorescence optics, showing green EG4 cells attached to a 8-cell stage embryo. $(\times 400)$

Fig 5.

a: EG4-GFP cells attached to a compacted 8-cell stage embryo, and a sandwich aggregate composed of two embryos and a group of EG4-GFP cells. (? 100)

b: The same field of Fig 5 a, but with fluorescence optics, showing green EG4 cells. $(\times 100)$

Fig 6.

a: The chimeric embryos developed to morulae or blastocysts after $30 \mathrm{~h}$ of aggregation, with regular optics. $(\times 100)$

b: The same field of Fig 6 a, but with fluorescence optics, showing green EG4 cells located in morulae (arrow head indicated) and inner cell masses of blastocysts (arrow indicated). (×100)

Fig 7.

The chimeric embryos in morula or blastocyst stage were developed from embryos with only 1-2 green cells attached. 1-6 green cells were observed in these chimeric embryos. One cell was seen in trophectoderm (upper right inset). $(\times 100)$ 
$\mathrm{Xu} \mathrm{X}$ et al.
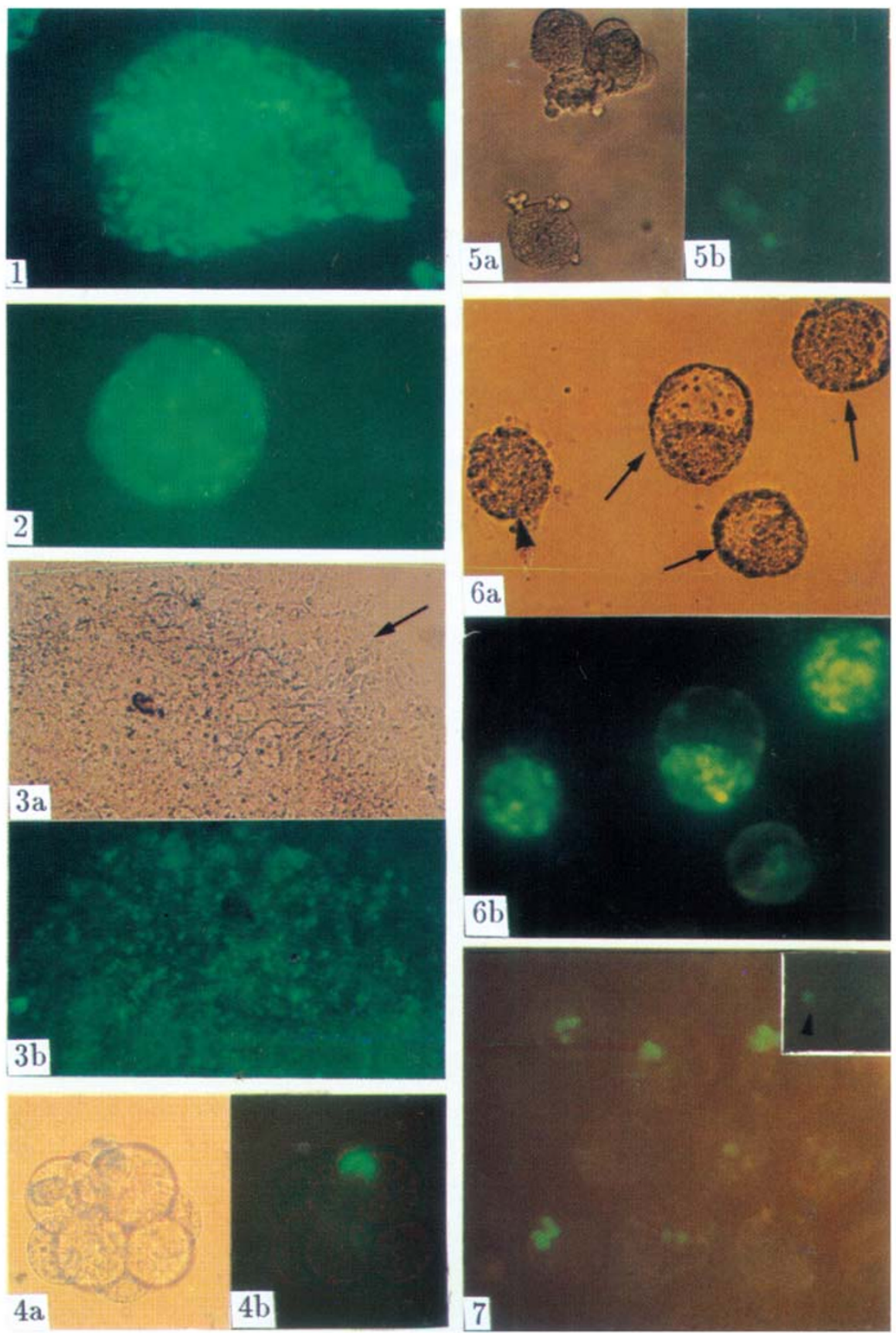

could be passaged many times in an undifferentiated state in the presence of LIF.

EG4-GFP cells developed to embryonic bodies after 3-4 d of suspension culture and exhibited bright green fluorescence under UV light (Fig 2). Differentiated cells with green fluorescence under UV light (Fig 3) grew out from the margin of embryonic bodies recultured on gelatin-treated 24 -well plate for 1-2 d in medium without LIF, suggesting 
The developmental fate of green fluorescent mouse embryonic germ cells in chimeric embryos

that EG4-GFP cells still retained typical characteristics of EG cells, such as morphology and differentiation capacity in vitro.

\section{Production of chimeric embryos by aggregation}

Groups of EG4-GFP cells were cocultured with 8-cell embryos in small holes in agar layer and after several hours of incubation, the adherence of cells to embryos was followed. Fig 4 showed the adhered EG4-GFP cells to a blastomere of a single 8-cell stage embryo, Fig 5 indicated the result from a group of EG4-GFP cells which was originally placed in the middle of two embryos just as sandwiches. After overnight culture, the majority of aggregates developed to morulae and blastocysts (Fig 6 ).

Through the analysis of the results of 128 embryos made in the present work, the following general conclusions can be arrived: 1) one embryo is enough for producing the chimeric embryo by aggregation, although sandwiches of embryo-cells-embryo can also develop normally in vitro; moreover, both compacted and uncompacted 8-cell stage embryos with attached EG4-GFP cells could form morphologically normal blastocysts after overnight culture. EG cells aggregated to uncompacted embryos tend to be located between blastomeres, while those aggregated to the compacted embryo could only remain beside the embryo in initial stage. It is indicated that EG4-GFP cells which were stayed outside of the embryo could also contribute to chimeric embryos. 2) The majority of EG4GFP cells could form a part of morula and ICM of blastocyst during in vitro culture. Green EG4 cells located in trophectoderm of blastocyst were occasionally observed (Fig 7 , upper right inset, arrowhead indicated), but the ratio of green cells located in inner cell mass and trophectoderm was not analyzed. 3) EG4-GFP cells attached to 8-cell stage embryos could still divide during blastocyst formation when a single or a couple of green cells were cocultured with each receipt embryo, 1-6 green cells were finally observable in blastocysts (Fig 7), indicating that some EG4-GFP cells did divide once or twice during the development of blastocysts.

We have transferred 19 chimeric blastocysts produced by aggregation to 2 foster mothers, but got no new borns. 9 chimeric blastocysts produced by microinjection of EG4GFP cells to perivellitine space of 8-cell stage embryos were transferred to 1 foster mother and 2 new borns were obtained. Five days after birth, one was killed and inspected. Tissues of spleen, brain and intestine were placed on slides with coverslip, pressed and followed by observation under UV light. Tissues of intestine showed obvious green fluorescence in some area (Fig 8 ), tissues of spleen showed less green fluorescence, while no green fluorescence was observed in brain. The result suggested that green EG4-GFP cells had contributed to some somatic tissues of that chimeric mouse.

LacZ labeled ES cells were extensively reported in previous studies, ES cells co-transfected with LacZ and a particular gene were used to produce transgenic mice, and expression patterns of that particular gene were screened for by histochemical staining of whole embryos for the reporter gene product ( $\beta$-galactosidase). LacZ as well as other invasive cell markers require substrate reactions to be visualized[11]. As a novel non-invasive cell 
$\mathrm{Xu} \mathrm{X}$ et al.
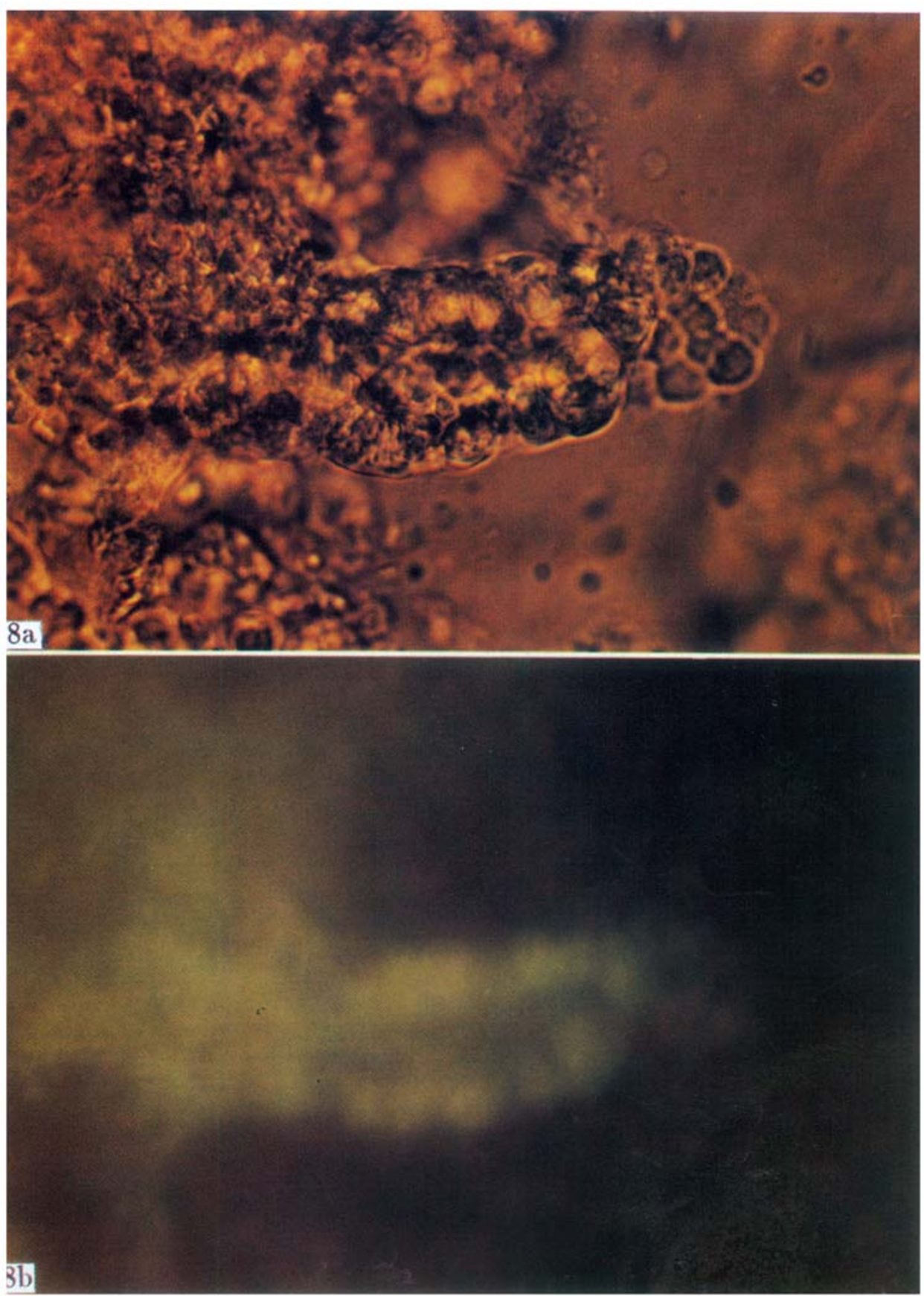

\section{Fig 8.}

a: Part of intestine from a 5-day-old chimeric mouse produced by microinjection of EG4-GFP cells to perivilline space of 8-cell-stage host embryos. $(\times 400)$

b: The same field of Fig 8 a, but with fluorescence optics, showing obvious green fluorescence in some area. $(\times 400)$ 
The developmental fate of green fluorescent mouse embryonic germ cells in chimeric embryos

marker, EGFP can be observed in real time in vitro and in situ in the transgenic animals and cell lineages. Nagy's group has established green ES cell lines and produced green fluorescent mice recently[12]. We generated successfully EG cell lines carrying EGFP as a marker gene, and made some preliminarty observations of the developmental fate of EG4-GFP cells in chimeric embryos. The result showed that EG4-GFP cells possessed the ability to take part in host embryo development.

\section{ACKNOWLEDGEMENTS}

This project was supported by grants of Chinese Academy of Sciences, National "Pan Deng” program and The Rockefeller Foundation. We are most grateful to Prof. Shi WeiKang for his valuable discussion on this work and we also thank Ms. Li Xiu Lan for her technical assistance.

\section{REFERENCES}

[1] Wylie CC and Heasman J. Migration, proliferation and potency of primordial germ cells. Seminar in Developmental Biology, 1993; 4:161-70.

[2] Matsui Y, Zsebo K, Hogan BLM. Derivation of pluripotential embryonic germ cells from murine primordial germ cells in culture. Cell 1992; 70:841-7.

[3] Resnick J L, Bixler LS, Cheng LZ, et al. Long term proliferation of mouse primordial germ cells in culture. Nature 1992; 359:550-1.

[4] Xu Xin, Tsung Hsiao-chien, Yan Yuan-chang. Establishment and differentiation of EG cells derived from primordial germ cells. Acta Biologiae Experimentalis Sinica, 1999: in press.

[5] Stewart CL, Gadi I, Bhatt H. Stem cells from primordial germ cells can reenter the germ line. Developmental Biology, 1994; 161:626-8.

[6] Labosky PA, Barlow DP, Hogan BLM. Mouse embryonic germ cell lines transmission through the germline and differences in the methylation imprint of insulin like growth factor 2 receptor gene compared with ES cell lines. Development 1994; 120:3197-204.

[7] Kim DW, Uetsuki T, Kaziro Y, et al. Use of the human elongation factor 1 a promotor as a versatile and efficient expression system. Gene 1990; 91:217-23.

[8] Bradley A. Production and analysis of chimaeric mice. In: Robertson EJ. eds. Teratocarcinomas and embryonic stem cells: a practical research, IRL press: Oxford 1987:113-51.

[9] Tsung Hsiao-chien and Mummery L. Effects of feeder layer and BRL conditioned medium on mouse embryonic stem cells. Cell Research 1990; 1:35-51.

[10]Saburi S, Azuma S, Sato E, et al. Developmental fate of single embryonic stem cells microinjected into 8-cell-stage mouse embryos. Differentiation, 1997; 62:1-11

[11]Ramirez-Solis R. and Bradley A. Advances in the use of embryonic stem cell technology. Current Opinion in Biotechnology, 1994; 5:528-33.

[12]Hadjantonakis AK, Gertsenstein M, Ikawa M, et al. Generating green fluorescent mice by germline transmission of green fluorescent ES cells. Mechanisms of Development, 1998; 76:79-90.

Received May-8-1999. Revised July-7-1999. Accepted July-24-1999. 